\title{
IOSS Control for a Class of Switched Hopfield Neural Networks with Disturbance Input
}

\author{
C.K. Ahn \\ School of Electrical Engineering, Korea University \\ 145, Anam-ro, Seongbuk-gu, Seoul, 136-701 Korea
}

\begin{abstract}
This paper is concerned with the input-output-to-state stable (IOSS) controller design for switched Hopfield neural networks with disturbance input. We propose a new set of linear matrix inequality (LMI) conditions to ascertain that switched Hopfield neural networks are IOSS based on a quadratic positive semi-definite function. Switched Hopfield neural networks without disturbance input are also asymptotically stable under an additional set of LMI conditions. The proposed IOSS controller can be determined by solving a set of LMIs, which can be checked by using existing numerical algorithms.
\end{abstract}

Keywords- input-output-to-state stable (IOSS) control; switched Hopfield neural network; disturbance input; linear matrix inequality (LMI) approach

\section{INTRODUCTION}

Over the past few decades, many research results on neural networks have been reported in the literature because of their practical advantages, such as learning ability, classification, parallel computation, and function approximation. Interesting applications of neural networks are combinatorial optimization, nonlinear control, pattern recognition, and other many areas. The applications mainly depend on the stability and robustness of neural networks [1]. For this reason, the analysis of the stability and robustness for neural networks has been one of important research topics. On the other hand, switched dynamical systems often arise when systems have abrupt changes. Switched systems have been researched extensively in the literature during the past decades [2, 3, 4, 5]. Recently, switched Hopfield neural networks have been extensively researched in the field of gene selection and high-speed signal processing [6, 7]. Some new research results on learning, filtering, and stability for switched Hopfield neural networks have been presented in $[8,9,10,11,12,13]$.

The input-output-to-state stability (IOSS) concept [14, 15] is a significant method for investigating several properties of complex dynamical systems with disturbances. The IOSS concept can deal with complex dynamical systems using the input-output characteristic. Thus, many research results on the IOSS have been reported in recent years [14, 15, 16, 17]. Now, we have the following question: is there an IOSS based controller for switched Hopfield neural networks with disturbance input? This paper provides an answer to the question. For the IOSS based control for switched Hopfield neural networks with disturbance input, there have been no papers published in the literature.

In this paper, we use the IOSS approach to obtain a new controller for switched Hopfield neural networks with disturbance input. This controller is called an input-output-to- state stable (IOSS) controller. A new set of linear matrix inequality (LMI) based conditions for the IOSS controller is proposed such that switched Hopfield neural networks are asymptotically stable and IOSS for disturbance input. The gain matrix of the IOSS controller can be determined by solving a set of LMIs $[18,19]$.

\section{IOSS CONTROL FOR SWITCHED HOPFIELD NEURAL NETWORKS}

Consider the following switched Hopfield neural network:

$$
\begin{aligned}
& \dot{x}(t)=A_{\chi} x(t)+W_{\chi} \theta(x(t))+u(t)+G_{\chi} d(t), \\
& y(t)=C_{\chi} x(t)+D_{\chi} d(t)
\end{aligned}
$$

where $x(t)=\left[x_{1}(t) \ldots x_{n}(t)\right]^{T} \in R^{n}$ is the state vector, $u(t)=\left[u_{1}(t) \ldots u_{n}(t)\right]^{T} \in R^{n}$ is the control input, $y(t)=\left[y_{1}(t) \ldots y_{p}(t)\right]^{T} \in R^{p} \quad$ is the output vector, $d(t)=\left[d_{1}(t) \ldots d_{k}(t)\right]^{T} \in R^{k}$ is the disturbance vector, $\chi$ is a switching signal which takes its values in the finite set $\mathrm{I}=\{1,2, \ldots, N\}, N$ is the number of Hopfield neural

networks, $A_{\chi}=\operatorname{diag}\left\{-a_{1, \chi}, \ldots,-a_{n, \chi}\right\} \in R^{n \times n}$ $\left(a_{i, \chi}>0, i=1, \ldots, n\right) \quad$ is the self-feedback matrix, $W_{\chi} \in R^{n \times p} \quad$ is the weight matrix, $\theta(x)=\left[\theta_{1}(x), \ldots, \theta_{p}(x)\right]^{T}: R^{n} \rightarrow R^{p}$ is the nonlinear sigmoid function vector with the Lipschitz constant $L_{\theta}>0$, and $C_{\chi} \in R^{p \times n}, D_{\chi} \in R^{p \times k}, G_{\chi} \in R^{n \times k}$ are known constant matrices. We assume that the instantaneous value of the switching signal $\chi$ is available in real-time. Define $\xi(t)=\left(\xi_{1}(t), \xi_{2}(t), \ldots, \xi_{N}(t)\right)^{T}$, where $\xi_{i}(t)=1$ when the $\chi$-th switched Hopfield neural network is selected and $\xi_{i}(t)=0$ otherwise. Based on the definition of $\xi(t)$, the switched Hopfield neural network (1)-(2) is represented by

$$
\dot{x}(t)=\sum_{i=1}^{N} \xi_{i}(t)\left[A_{i} x(t)+W_{i} \theta(x(t))+u(t)+G_{i} d(t)\right],
$$




$$
y(t)=\sum_{i=1}^{N} \xi_{i}(t)\left[C_{i} x(t)+D_{i} d(t)\right]
$$

where $\xi(t)$ satisfies $\sum_{i=1}^{N} \xi_{i}(t)=1$.

Definition $1[14,15]$ A function $\gamma: R_{\geq 0} \rightarrow R_{\geq 0}$ is a $\mathrm{K}$ function if it is continuous, strictly increasing and $\gamma(0)=0$. It is $a \mathrm{~K}_{\infty}$ function if it is a $\mathrm{K}$ function and $\gamma(s) \rightarrow \infty$ as $s \rightarrow \infty$. A function $\beta: R_{\geq 0} \times R_{\geq 0} \rightarrow R_{\geq 0}$ is a KL function if, for each fixed $t \geq 0$, the function $\beta(\cdot, t)$ is a $\mathrm{K}$ function, and for each fixed $s \geq 0$, the function $\beta(s, \cdot)$ is decreasing and $\beta(s, t) \rightarrow 0$ as $t \rightarrow \infty$.

The purpose of this paper is to design a new stabilization controller of the form $u(t)=K x(t)\left(K \in R^{n \times n}\right)$ such that the switched Hopfield neural network (3)-(4) satisfies

$$
\mathrm{P} x(t) \mathrm{P} \leq \max \left\{\beta(\mathrm{P} x(0) \mathrm{P}, t), \gamma_{1}\left(\sup _{0 \leq \tau \leq t} \mathrm{P} d(\tau) \mathrm{P}\right), \gamma_{2}\left(\sup _{0 \leq \tau \leq t} \mathrm{P} y(\tau) \mathrm{P}\right)\right\}
$$

for $d(t) \neq 0$, where $\gamma_{i}(s)(i=1,2)$ is a $\mathrm{K}$ function and $\beta(s, t)$ is a $\mathrm{K} L$ function.

A set of LMI conditions for the IOSS control of the switched Hopfield neural network (3)-(4) is proposed in the following theorem:

Theorem 1 Assume that there exist common matrices $P=P^{T}>0, S_{1}=S_{1}^{T}>0, S_{2}=S_{2}^{T}>0, S_{3}=S_{3}^{T}>0$, and $Y$ such that

$$
\left[\begin{array}{ccc}
\Theta_{i} & P G_{i}-C_{i}^{T} S_{2} D_{i} & P W_{i} \\
G_{i}^{T} P-D_{i}^{T} S_{2} C_{i} & -D_{i}^{T} S_{2} D_{i}-S_{3} & 0 \\
W_{i}^{T} P & 0 & -I
\end{array}\right]<0, i=1,2, \ldots, N,
$$

where $\Theta_{i}=\left(P A_{i}+Y\right)^{T}+P A_{i}+Y+L_{\phi}^{2} I-C_{i}^{T} S_{2} C_{i}+S_{1}$. Then, the switched Hopfield neural network (3)-(4) is IOSS. The IOSS controller is given by $u(t)=P^{-1} Y x(t)$.

Proof. We obtain the following closed-loop switched Hopfield neural network with the controller $u(t)=K x(t)$ :

$$
\dot{x}(t)=\sum_{i=1}^{N} \xi_{i}(t)\left[\left(A_{i}+K\right) x(t)+W_{i} \theta(x(t))+G_{i} d(t)\right] .
$$

Consider a positive semi-definite function: $V(t)=x^{T}(t) P x(t)$, which satisfies the Rayleigh inequality [20]:

$$
\alpha_{1}(\mathrm{P} x(t) \mathrm{P}) \leq V(t) \leq \alpha_{2}(\mathrm{P} x(t) \mathrm{P}),
$$

where $\alpha_{1}(r) @ \lambda_{\text {min }}(P) r^{2}, \alpha_{2}(r) @ \lambda_{\text {max }}(P) r^{2}$, and $\lambda_{\text {min }}(\cdot)$ and $\lambda_{\text {max }}(\cdot)$ are the maximum and minimum eigenvalues of the matrix. Note that $\alpha_{1}(r)$ and $\alpha_{2}(r)$ are
$\mathrm{K}_{\infty}$ functions. Using the Young's inequality [20], $V(t)$ satisfies

$$
\begin{gathered}
\dot{V}(t)=\sum_{i=1}^{N} \xi_{i}(t)\left\{x^{T}(t)\left[A_{i}^{T} P+K^{T} P+P A_{i}+P K\right] x(t)+x^{T}(t) P W_{i} \theta(x(t))\right. \\
\left.+\theta^{T}(x(t)) W_{i}^{T} P x(t)+x^{T}(t) P G_{i} d(t)+d^{T}(t) G_{i}^{T} P x(t)\right\} \\
\leq \sum_{i=1}^{N} \xi_{i}(t)\left\{x^{T}(t)\left[A_{i}^{T} P+K^{T} P+P A_{i}+P K+L_{\phi}^{2} I+P W_{i} W_{i}^{T} P\right] x(t)\right. \\
\left.+x^{T}(t) P G_{i} d(t)+d^{T}(t) G_{i}^{T} P x(t)\right\} .
\end{gathered}
$$$$
\text { Adding the zero equality }
$$$$
y^{T}(t) S_{2} y(t)-\sum_{i=1}^{N} \xi_{i}(t)\left[C_{i} x(t)+D_{i} d(t)\right]^{T}
$$$$
S_{2}\left[C_{i} x(t)+D_{i} d(t)\right]=0 \text { to }(9) \text {, we obtain }
$$

$$
\begin{gathered}
\dot{V}(t) \leq \sum_{i=1}^{N} \xi_{i}(t)\left\{x ^ { T } ( t ) \left[A_{i}^{T} P+K^{T} P+P A_{i}+P K+L_{\phi}^{2} I+P W_{i} W_{i}^{T} P\right.\right. \\
\left.-C_{i}^{T} S_{2} C_{i}\right] x(t)-d^{T}(t) D_{i}^{T} S_{2} D_{i} d(t)+x^{T}(t)\left[P G_{i}-C_{i}^{T} S_{2} D_{i}\right] d(t) \\
\left.+d^{T}(t)\left[G_{i}^{T} P-D_{i}^{T} S_{2} C_{i}\right] x(t)+y^{T}(t) S_{2} y(t)\right\} \\
=\sum_{i=1}^{N} \xi_{i}(t)\left[\begin{array}{c}
x(t) \\
d(t)
\end{array}\right]^{T}\left[\begin{array}{cc}
(1,1)_{i} & P G_{i}-C_{i}^{T} S_{2} D_{i} \\
G_{i}^{T} P-D_{i}^{T} S_{2} C_{i} & -D_{i}^{T} S_{2} D_{i}-S_{3}
\end{array}\right]\left[\begin{array}{c}
x(t) \\
d(t)
\end{array}\right] \\
-x^{T}(t) S_{1} x(t)+y^{T}(t) S_{2} y(t)+d^{T}(t) S_{3} d(t), \quad(10) \\
\text { where } \\
(1,1)_{i}=A_{i}^{T} P+K^{T} P+P A_{i}+P K+L_{\phi}^{2} I+P W_{i} W_{i}^{T} P-C_{i}^{T} S_{2} C_{i}+S_{1} .
\end{gathered}
$$

Define

$$
\Psi_{i}=A_{i}^{T} P+K^{T} P+P A_{i}+P K+L_{\phi}^{2} I-C_{i}^{T} S_{2} C_{i}+S_{1} .
$$

The following condition:

$$
\left[\begin{array}{ccc}
\Psi_{i} & P G_{i}-C_{i}^{T} S_{2} D_{i} & P W_{i} \\
G_{i}^{T} P-D_{i}^{T} S_{2} C_{i} & -D_{i}^{T} S_{2} D_{i}-S_{3} & 0 \\
W_{i}^{T} P & 0 & -I
\end{array}\right]<0, i=1,2, \ldots, N,
$$

which is equal to

$$
\left[\begin{array}{cc}
(1,1)_{i} & P G_{i}-C_{i}^{T} S_{2} D_{i} \\
G_{i}^{T} P-D_{i}^{T} S_{2} C_{i} & -D_{i}^{T} S_{2} D_{i}-S_{3}
\end{array}\right]<0, i=1,2, \ldots, N,
$$

implies

$$
\begin{aligned}
& \dot{V}(t)<-x^{T}(t) S_{1} x(t)+y^{T}(t) S_{2} y(t)+d^{T}(t) S_{3} d(t) \\
& \leq-\lambda_{\text {min }}\left(S_{1}\right) \mathrm{P} x(t) \mathrm{P}^{2}+\lambda_{\text {max }}\left(S_{2}\right) \mathrm{P} y(t) \mathrm{P}^{2}+\lambda_{\text {max }}\left(S_{3}\right) \mathrm{P} d(t) \mathrm{P}^{2} \\
& =-\alpha_{3}(\mathrm{P} x(t) \mathrm{P})+\alpha_{4}(\mathrm{P} y(t) \mathrm{P})+\alpha_{5}(\mathrm{P} d(t) \mathrm{P}),
\end{aligned}
$$

where $\alpha_{3}(r) @ \lambda_{\text {min }}\left(S_{1}\right) r^{2}, \alpha_{4}(r) @ \lambda_{\text {max }}\left(S_{2}\right) r^{2}$, and $\alpha_{5}(r) @ \lambda_{\max }\left(S_{3}\right) r^{2}$. Note that $\alpha_{3}(r), \alpha_{4}(r)$, and $\alpha_{5}(r)$ are $\mathrm{K}_{\infty}$ functions. According to [14, 15], $V(t)$ is an IOSSLyapunov function in view of (8) and (15). Introducing a change of variable $P K=Y$, (11) is equal to the LMI (6). Then, $K=P^{-1} Y$. This completes the proof. $\square$ 
Corollary 1 Assume that there exist common matrices $P=P^{T}>0, S_{1}=S_{1}^{T}>0, S_{2}=S_{2}^{T}>0, S_{3}=S_{3}^{T}>0$, and $Y$ such that

$$
\begin{aligned}
& {\left[\begin{array}{ccc}
\Theta_{i} & P G_{i}-C_{i}^{T} S_{2} D_{i} & P W_{i} \\
G_{i}^{T} P-D_{i}^{T} S_{2} C_{i} & -D_{i}^{T} S_{2} D_{i}-S_{3} & 0 \\
W_{i}^{T} P & 0 & -I
\end{array}\right]<0,} \\
& S_{1}-C_{i}^{T} S_{2} C_{i}>0,
\end{aligned}
$$

for $i=1,2, \ldots, N$. Then, the switched Hopfield neural network (3)-(4) with the controller $u(t)=P^{-1} Y x(t)$ is IOSS for $d(t) \neq 0$. Moreover, it is asymptotically stable for $d(t)=0$.

Proof. Using Theorem 1, the LMI condition in Theorem 1 ascertains that the switched Hopfield neural network (3)-(4) with the controller $u(t)=P^{-1} Y x(t)$ for $d(t) \neq 0$ is IOSS. When $d(t)=0$, it is observed from (13) and (4) that

$$
\begin{gathered}
\dot{V}(t)<-\sum_{i=1}^{N} \xi_{i}(t) x^{T}(t)\left[S_{1}-C_{i}^{T} S_{2} C_{i}\right] x(t) \\
\leq-\sum_{i=1}^{N} \xi_{i}(t) \lambda_{\min }\left(S_{1}-C_{i}^{T} S_{2} C_{i}\right) \mathrm{P} x(t) \mathrm{P}^{2} .
\end{gathered}
$$

(17) implies $\dot{V}(t)<0$, which guarantees the asymptotic stability from Lyapunov stability theory. This completes the proof. $\square$

\section{CONCLUSION}

This paper has proposed the IOSS controller for switched Hopfield neural networks with disturbance input. A new set of sufficient LMI conditions was established to guarantee that switched Hopfield neural networks are asymptotically stable and IOSS for disturbance input. The proposed IOSS controller was obtained by solving a set of LMIs. The proposed control method can be extended to a general class of switched nonlinear systems with disturbances.

\section{REFERENCES}

[1] M. M. Gupta, L. Jin, and N. Homma. Static and Dynamic Neural Networks. Wiley-Interscience, 2003.

[2] N.H. Farra, P. Mhaskar, and P.D. Christofides. Output feedback control of switched nonlinear systems using multiple Lyapunov functions. Systems \& Control Letters, 54 (2005) 1163-1182.

[3] Y. Zhang, X.Z. Liu, and X.M. Shen. Stability of switched systems with time delay. Nonlinear Analysis: Hybrid Systems, 1 (2007) 44-58.

[4] G.D. Zong, S.Y. Xu, and Y.Q. Wu. Robust stabilization for uncertain switched impulsive control systems with state delay: an LMI approach. Nonlinear Analysis: Hybrid Systems, 2 (2008) 1287-1300.

[5] P. Niamsup. Stability of time-varying switched systems with timevarying delay. Nonlinear Analysis: Hybrid Systems, 3 (2009) 631-639.

[6] Y. Tsividis. Switched neural networks. United States Patent, Patent number 4873661, 1989.

[7] T.X. Brown. Neural networks for switching. IEEE Communications Magazine, (1989) 72-81.
[8] C. K. Ahn. Passive learning and input-to-state stability of switched Hopfield neural networks with time-delay. Information Sciences, 180(23):4582-4594, 2010.

[9] C. K. Ahn. Exponentially convergent state estimation for delayed switched recurrent neural networks. European Physical Journal E, 34(11):122, 2011.

[10] C. K. Ahn. Linear matrix inequality optimization approach to exponential robust filtering for switched Hopfield neural networks. Journal of Optimization Theory and Applications, 154(2):573-587, 2012

[11] C. K. Ahn. An error passivation approach to filtering for switched neural networks with noise disturbance. Neural Computing and Applications, 21(5):853-861, 2012.

[12] C. K. Ahn. Switched exponential state estimation of neural networks based on passivity theory. Nonlinear Dynamics, 67(1):573-586, 2012.

[13] C.K. Ahn and Y.S. Lee. Sets of generalized exponential stability criteria for switched multilayer dynamic neural networks. Advances in Difference Equations, 2012(150):1-8, 2012.

[14] E.D. Sontag and Y. Wang. Output-to-state stability and detectability of nonlinear systems. Syst. Contr. Lett., 29:279-290, 1997.

[15] M. Krichman, E.D. Sontag, and Y. Wang. Input-output-to-state stability. SIAM J. Control Optim., 39:1874-1928, 2001.

[16] D.S. Laila and D. Nesic. Changing supply rates for input-output to state stable discrete-time nonlinear systems with applications. Automatica, 39:821-835, 2003.

[17] C. Cai and A.R. Teel. Asymptotic characterizations of input/output-tostate stability for discrete-time systems. Syst. Contr. Lett., 56:408-415, 2007.

[18] S. Boyd, L. E. Ghaoui, E. Feron, and V. Balakrishinan. Linear matrix inequalities in systems and control theory. SIAM, Philadelphia, PA, 1994.

[19] P. Gahinet, A. Nemirovski, A. J. Laub, and M. Chilali. LMI Control Toolbox. The Mathworks Inc., 1995.

[20] G. Strang. Introduction to Applied Mathematics. Wellesley Cambridge Press, Cambridge, 1986. 